\title{
The North Laine Shopping Guide: A Case Study in Modelling Trust in Applications
}

Jon Robinson, Ian Wakeman, Dan Chalmers, and Anirban Basu

\begin{abstract}
Facilitating navigation through commercial spaces by third party systems is a likely step in pervasive computing. For these applications to fully engage people they must build trust relationships in a natural manner. We hypothesize that the use of an explicit trust model in the design of the application would improve the rate at which trust is generated. To investigate this hypothesis, we have taken as a case study the design of a shopping guide for a local trading association. We have created an explicit trust model and incorporated this into our design. We have evaluated both our model and our application. The results of this confirmed our hypothesis and provided additional insight into how to model trust in the design of applications.
\end{abstract}

\section{Introduction}

We are moving towards a time when the environment around us is full of computing applications, most of which will be owned by third parties with whom we have no direct relationship. These applications will offer services such as providing navigational support through shopping arcades or reviews of the available amenities. For these applications to fully engage people, they must quickly build trust between users and the services they provide. The object of trust in this context is both the application and the service provider behind the application.

\author{
Jon Robinson \\ Department of Informatics, University of Sussex, UK, e-mail: J.R.Robinson@ sussex.ac.uk \\ Ian Wakeman \\ Department of Informatics, University of Sussex e-mail: ianw@sussex.ac.uk \\ Dan Chalmers \\ Department of Informatics, University of Sussex e-mail: dc52@sussex.ac.uk \\ Anirban Basu \\ Department of Informatics, University of Sussex e-mail: ab25@sussex.ac.uk
}

Please use the following format when citing this chapter:

Robinson, J., Wakeman, I., Chalmers, D. and Basu, A., 2008, in IFIP International Federation for Information Processing, Volume 263; Trust Management II; Yücel Karabulut, John Mitchell, Peter Herrmann, Christian Damsgaard Jensen; (Boston: Springer), pp. $183-197$. 
The question arises about how to build applications which facilitate these trust relationships. We hypothesis that the use of an explicit trust model in the design of the application would improve the rate at which trust is generated. To investigate this hypothesis, we have taken as a case study the design of a shopping guide for a local trading association. We have investigated how to create an explicit trust model and how to incorporate this into our design where we only consider honest and non-malicous user feedback. We have then evaluated this model against the existing shopping guide which confirmed our hypothesis and provided additional insight into how to model trust in the design of applications.

Our contributions are to show how to create and make explicit a trust model within the design and to demonstrate how an explicit trust model improves applications.

In section 2 we examine the state of the art in trust models in consumer behaviour and e-commerce. Building on these models, we outline our trust model in section 3. We describe how we verify our trust model in section 3.1. In section 4 we discuss the implementation of the trust model within an application context. We provide an analysis of the trust building model and application in section 5. Finally, we conclude with a summary of our contribution in 6 .

\title{
2 Consumer behaviour based models of trust
}

\author{
Consumer behaviour can be defined as [1]: \\ the behaviour that consumers exhibit in searching for, purchasing, using, evaluating and \\ disposing of products, services and ideas which they expect will satisfy their needs.
}

The Consumer Behaviour literature distinguishes between the behaviour of individuals and organisations. While organisational models such as the Webster-Wind Model of Organisational Buying Behaviour [11] and the Sheth Model Model of Industrial Buying Behaviour [12] can provide insight into model creation, our domain of interest is on an individual consumer, and we have investigated these models in greater detail.

Whilst there are a number of notable examples of consumer behaviour models available (Howard-Sheth Model of Buyer Behaviour [7], Engel-Kollat-Blackwell Model [14] and Sheth Model of family decision making [13]) the commonly accepted standard of understanding individual motivations for consumptions is the purchase decision process [1].

The purchase decision process provides us with a means to distinctly classify the tasks involved within the decision making process of a consumer. These tasks are:

- Problem recognition: when a consumer realises that there is some need for an item.

- Information Search: when the consumer finds additional information regarding the item. This can be based on previous experience (internal) or on external searches based on hunting for information from a variety of sources. 
- Evaluation of alternatives: when the consumer has found a number of alternative brands, they evaluate each of the alternatives using the information at hand.

- Purchase: when the purchase of the item takes place once they have chosen a retailer and payment method.

- Post-Purchase evaluation: this happens after the purchase and is when the consumer decides if the purchase was successful or not.

- Divestment: the disposal of the product once they have finished using it.

We defer discussion of how these are integrated within our trust model until section 4. The final aspect regarding the divestment of the purchased item is not needed within our trust model as we are primarily interested in the processes leading up to this stage.

When exploring the literature for consumer behaviour, the notion of perceived risk was common place and acted as a central tenet for trust building systems in the e-commerce world. Perceived risk is based on the judgment that a consumer has on the severity of risk involved within a transaction. This is an important issue to take into consideration when designing a trust model as this can have a direct impact on how well the consumer trusts the information provided by our proposed model.

The literature identifies a number of different attributes that can affect the trust and perceived risk between a consumer and seller: benevolence; integrity; competence/ability; and predictability. Upon further inspection, these can be broken down to a number of trust attributes which we can use as the measurable attributes in our trust model as shown in table 1.

\begin{tabular}{|c|c|c|}
\hline availability & consistency & discretion \\
\hline fairness & loyalty & openness \\
\hline promise fulfillment & receptivity & trustworthy intentions \\
\hline intention to deliver & reputation & size \\
\hline willingness to customize & trustee's promises & motives \\
\hline moral integrity & goodwill & seals \\
\hline re-embedding & web site design & professionalism \\
\hline product reviews & price comparisons & \\
\hline
\end{tabular}

Table 1 Perceived Trust Attributes

The e-commerce literature also uses these models as a foundation for their discussion of trust models. For instance, Chen and Dhillon [2] propose the Path Model in which factors regarding the consumer, seller (in this case a firm), website and interactions act as inputs to the trust building attributes classified as competence, integrity and benevolence (which are commonly cited by others) to form a notion of overall trust which impacts on the consumers purchase decision.

Kim and Ahm [6] propose the Buyers Trust model where trust is formed through the transactions of market-makers and sellers based on a number of antecedents of trust for either. These are based on size, reputation, usability and security for the market-maker while expertise and reputation form the basis for a seller. 
McKnight et al [3] propose the Trust Building Model where trust is formed out of a set of antecedent factors (in this case those that deal with the sellers perceived reputation and site quality) and factors that deal with structural constraints of the infrastructure or institution (such as the perceived risk of the web). These factors determine the level of trust the consumer has in the seller. This dictates the behaviour of the consumer and how trusting they are in following the seller's advice, allowing the seller access to personal information or how it impacts on their decision to purchase from the seller.

Lee and Turban [10] propose the Model for Consumers' Trust in Internet Shopping where factors regarding the trustworthiness of the seller (in this case a merchant on the Internet). Their model uses ability, integrity and benevolence coupled with trustworthiness of the medium in which the transaction takes place. These are supplemented with a set of contextual factors that focus on the security and effectiveness of third parties to produce a set of scores (trust propensities) which form the basis of the overall trust allocated to the operation.

Riegelsberger et al [8] provide a number of ways in which trust can be enhanced between a consumer and seller. They discuss how seals can be used as a way for identifying the authenticity of sellers (i.e. a seal could be used which allows the customer to be confident that they can trust the seller) to interacting with a human presence instead of a web page and how important the role of the interface and subsequent professional issues that arise are in the process of forming a trusting relationship. They also go on in [9] to outline a framework between interactions of a user and system and how trust can be formed based on a set of contextual and intrinsic properties.

\section{Developing an application specific trust model}

We are a building a kiosk system (using a touch screen connected to a PC) to help customers choose a route visiting shops through an area of Brighton called the North Laine. The North Laine is a popular local shopping area consisting of over 380 shops spread over an area of approximately $0.75 \mathrm{~km}$ square. Customers should be able to enter the goods they are looking for in order to identify appropriate shops and then to refine these according to their preferences. The "best" (as defined through our trust model) shops identified will be formed into a route through the area, limited by the time available for shopping.

Our approach to building trust has been to incorporate aspects of the consumer purchase buying model along with a number of criteria which we have identified as having an impact on a person's decision. The resulting trust model is shown in figure 1 .

In accordance with the consumer behaviour model we have incorporated five main steps within our trust model:

- Problem Recognition: identifying which item or items the user is initially interested in purchasing. 


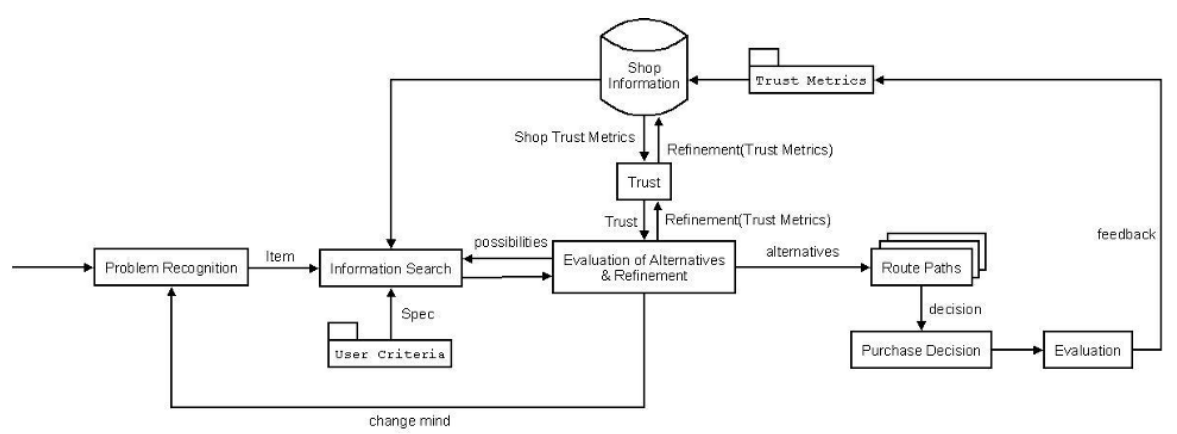

Fig. 1 The Trust Model

- Information Search: The process of considering what is available against the user's requirements. Additional information, such as the type of item, type of shop and the maximum distance to be traveled can be provided by the user to hone the results.

- Evaluation: The main interaction with our trust model occurs at this point. Based on the feedback given by previous users, the suggested shops will be ranked. This ranking process is discussed in more detail in section 4 . The user would be able to decide at this point which shops to visit, and thus plan a route path.

- Route Paths / Purchase Decision: The user goes shopping.

- Post-Purchase Evaluation (feedback): Once the shopping expedition has been completed, the post-purchase evaluation stage provides the user with an opportunity to leave feedback regarding their shopping experience. This would be in the form of rating the experience based on each of the trust metrics which in turn would be used to update the trust model.

We identified 23 candidate criteria for our trust model by traversing the consumer behaviour and e-commerce literature. We also added more attributes to the ones identified in table 1 which were domain specific (i.e. quality, aftesales and satisfaction). We then gathered questionnaire data from 15 students about recent shopping excursions, including both good and bad experiences. Statistical analysis identified which criteria were strongly correlated allowing us to remove unnecessary criteria. We then verified our criteria using data from the Hardens Restaurant Guide [15]. This resulted in the nine attributes that have been chosen for our trust model:

- Availability: The availability of the item.

- Quality: How good the item was.

- Reputation: The reputation of the shop that the item was bought from.

- After Sales: How good the after sales provided by the shop was if the customer had to return to the shop for any reason.

- Discretion: How well confidential details (for example credit card details) were handled.

- Satisfaction: How satisfied the customer was with the item that they bought. 
- Experience: How the customer would rate the shopping experience.

- Price: How well the price compares with others.

- Distance: How far the customer is willing to travel to a particular shop.

\subsection{Verifying the Trust Model}

To validate the trust attributes and to gather additional information about shopping habits, we produced a web-based questionnaire. The questionnaire had 164 participants start, of which 103 were fully completed. The purpose of the questionnaire was three fold:-

- gather details about the habits and demographics of the people shopping in the North Laine;

- provide "real" data to populate our system;

- and finally, to verify our trust model with data provided by the target users.

One of the key requirements of the study was to determine the relevance of each of the trust attributes. Participants were given the opportunity to rank the importance of each attribute using a range of scores from 1 (very important) to 5 (not important). The results are summarised in table 2 .

\begin{tabular}{|c|c|c|c|c|c|}
\hline & 1(Very Important) & 2 & 3 & 4 & 5 (Not important/I don't know) \\
\hline Quality & $40.8 \%$ & $32.0 \%$ & $16.5 \%$ & $5.8 \%$ & $4.9 \%$ \\
\hline Price & $36.9 \%$ & $37.9 \%$ & $15.5 \%$ & $4.9 \%$ & $4.9 \%$ \\
\hline Experience & $11.7 \%$ & $20.4 \%$ & $26.2 \%$ & $22.3 \%$ & $19.4 \%$ \\
\hline Distance & $3.9 \%$ & $15.5 \%$ & $28.2 \%$ & $26.2 \%$ & $26.2 \%$ \\
\hline Satisfaction & $41.7 \%$ & $45.6 \%$ & $9.7 \%$ & $1.9 \%$ & $1.0 \%$ \\
\hline Discretion & $7.8 \%$ & $7.8 \%$ & $19.4 \%$ & $20.4 \%$ & $44.7 \%$ \\
\hline After Sales & $7.8 \%$ & $12.6 \%$ & $22.3 \%$ & $24.3 \%$ & $33.0 \%$ \\
\hline Availability & $26.2 \%$ & $35.9 \%$ & $13.6 \%$ & $13.6 \%$ & $10.7 \%$ \\
\hline Reputation & $12.6 \%$ & $27.2 \%$ & $20.4 \%$ & $20.4 \%$ & $19.4 \%$ \\
\hline
\end{tabular}

Table 2 Importance of trust attributes

The results in table 2 indicate that each trust attribute is at least somewhat important (score 1-3) to a significant proportion of people (35-97\%). This indicates that each is important enough to justify its inclusion. No comments suggesting further attributes were made by any of the participants.

Because different people have differing interpretations of which attributes are important our model should provide a stronger sense of trust than a single summary rating the formative factors are clearly exposed.

A follow up question involved asking participants whether there were alternatives to the shops that they were they had visited (of which $48.5 \%$ said there were) and how these alternaitves fared in comparison based on each trust attribute. The results gained are summarised in table 3 . 


\begin{tabular}{|c|c|c|c|}
\hline & Others Worse & Very Similar & Others Better, but less important \\
\hline Quality & $23.2 \%$ & $69.6 \%$ & $7.1 \%$ \\
\hline Price & $37.5 \%$ & $48.2 \%$ & $14.3 \%$ \\
\hline Experience & $22.2 \%$ & $64.8 \%$ & $13.0 \%$ \\
\hline Distance (or time) & $26.8 \%$ & $62.5 \%$ & $10.7 \%$ \\
\hline Satisfaction & $27.3 \%$ & $65.5 \%$ & $7.3 \%$ \\
\hline Discretion & $7.4 \%$ & $81.5 \%$ & $11.1 \%$ \\
\hline After Sales & $10.9 \%$ & $69.1 \%$ & $20.0 \%$ \\
\hline Availability & $32.7 \%$ & $52.7 \%$ & $14.5 \%$ \\
\hline Reputation & $18.9 \%$ & $64.2 \%$ & $17.0 \%$ \\
\hline
\end{tabular}

Table 3 Rating of alternatives

One of the most interesting aspects of the results in table 3 is that one third of the respondents did not consider it important if there were other shops which had better alternatives. From these results we can draw the conclusion that the model can be verified by as there was no dominant attribute and all attributes were relevant.

\section{Design and Implementation}

When examining the e-commerce world, we found that the perceived risk between the user and application gave rise to a number of issues to be addressed within our design of the kiosk system. For example, we have previously mentioned the notion of professionalism with respect to the interface acting as a trust building factor, separate from the trust model, that can positively impact on the user experience.

Some of these factors are not directly relevant to the kiosk application. For instance, the factors that deal with financial information would not be used within the system, but instead could form a logical association with one or more of the trust attributes, while personally identifiable information would not have any direct impact on the system. Similarly, we have not explored novel HCI approaches, the use of avatars, gestural input etc.

Egger [5] provides a trust model that incorporates pre-purchase knowledge (or in our case, the information gathering stage, to determine the item to purchase), interface properties and informational content which are aspects of the user interface that they base their trust model on. However, for our purposes, a more in-depth trust model is required which will drive the informational content within the application.

There is a growing body of literature which discusses trust in kiosk systems. However, these focus on the security aspects of trust, for instance in e-voting where the trust is used in authenticating that the user is who they say they are by consulting an authentication server, or by providing trust so that the user is confident in the handling of financial data (e.g. payment through credit cards). However, we have yet to find a kiosk system that incorporates a trust model within their design in the same way that we propose. 
Our trust model is founded upon the consumer behaviour model and as such there are distinct phases in which the user must traverse when interacting with our kiosk. The first stage deals with the information gathering aspect of the model. Here, the user is provided with a set of categories of shops from which to choose the type of item that they are interested in. However, as the number of categories can be quite expansive, the user is provided with a more focused view of the types of shops that can be chosen from. This view provides a finer grained selection process which allows the user to identify shops selling a particular item for which they are interested in purchasing. Figure 2 highlights this process.
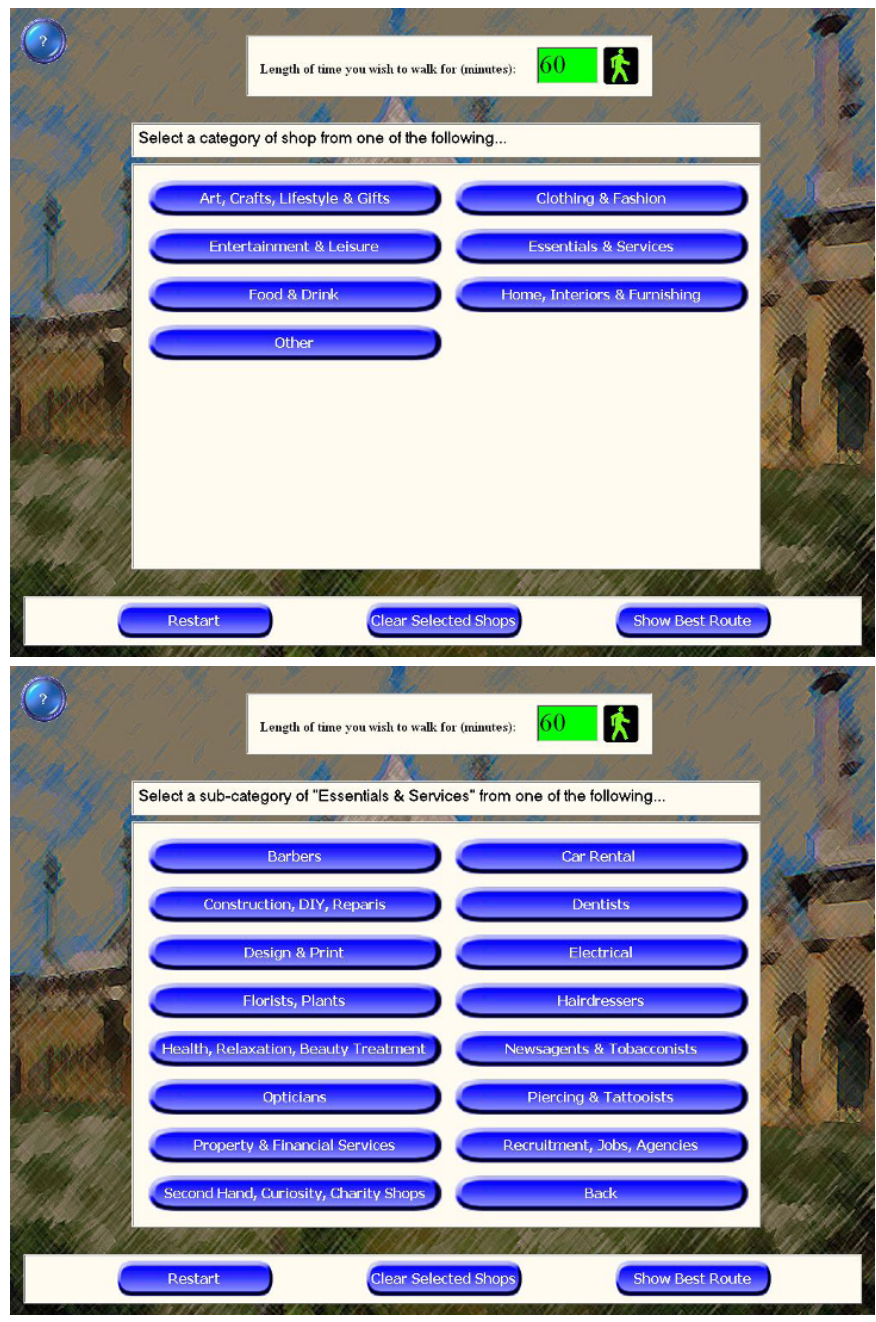

Fig. 2 Information Gathering Stage 


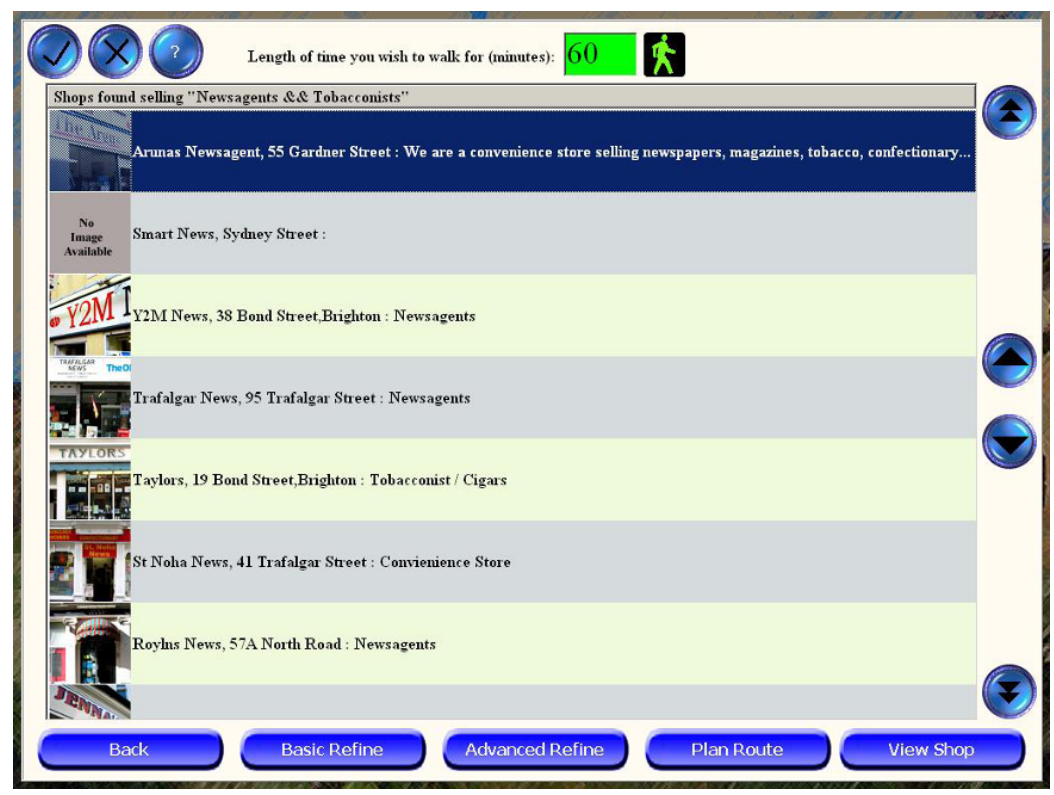

Fig. 3 Evaluation and Refinement Stage

Once the user has decided what type of item they are interested in purchasing the kiosk will present them with the shops that best match their requirements. Figure 3 shows the evaluation stage of the interface. It is at this stage that the trust model manifests itself within the user interface. The trust model uses as a measurable foundation, a set of nine trust attributes (see section 4) which are provided by previous peoples shopping experiences. The system calculates (using the following steps) a set of dynamic correlations between each of the trust attributes which forms the ranking order for the shops that are to be displayed.

1. For each attribute, work out the correlation between it and another to form a matrix of correlation co-efficients (Pearson Product-Moment Correlation) comparisons.

2. Filter out any duplicates to leave distinct correlations between all attributes.

3 . Based on this set of correlations, order the attribute correlations depending on $\operatorname{high}(0.5$ to 1.0$)$, medium( 0.3 to 0.49$)$ and $\operatorname{low}(0$ to 0.29$)$ values to form a weighting.

4. The value of each of the attribute correlations for each shop is calculated based on the weightings found in step (3).

- Any refinement of a trust attributes range is taken into account at this point. Rating scores that fall outside of the specified range are ignored. Based on this refined set of ratings, the overall value corresponding to the correlations for the shop are calculated. 
5. Using values based on the weightings of the trust attributes combinations given in step (4), the shops are ordered from highest to lowest based on the summation of all weightings values.

As the trust model is dynamic, the correlations between each of the trust attributes and their final relationship to how results are order can change over time. This provides the model with a high degree of flexibility, rather than pre-determining which correlations to use before hand.

The shops that are presented to the user are thus ranked in order based on these correlated rankings, such that those shops with a higher ranking will appear at the top of the presented shops. As the more favorable shops are now shown to the user, they have a higher chance of being chosen instead of ones with a lower ranking, and thus further down the list of presented shops.

At this stage the user can accept what is presented and make their selection, or they can refine the shops that have been provided by the system. There are two ways in which a user can refine the trust model. The first is through directly altering the influence of each attribute on the results extracted from the model. This would be then be reflected in the restricted set of shops provided in the evaluation stage where those shops with ratings that fall outside of the selected ranges will not be shown. This would also impact on the correlations and resulting rankings of attributes and consequently the ordering of the shops.

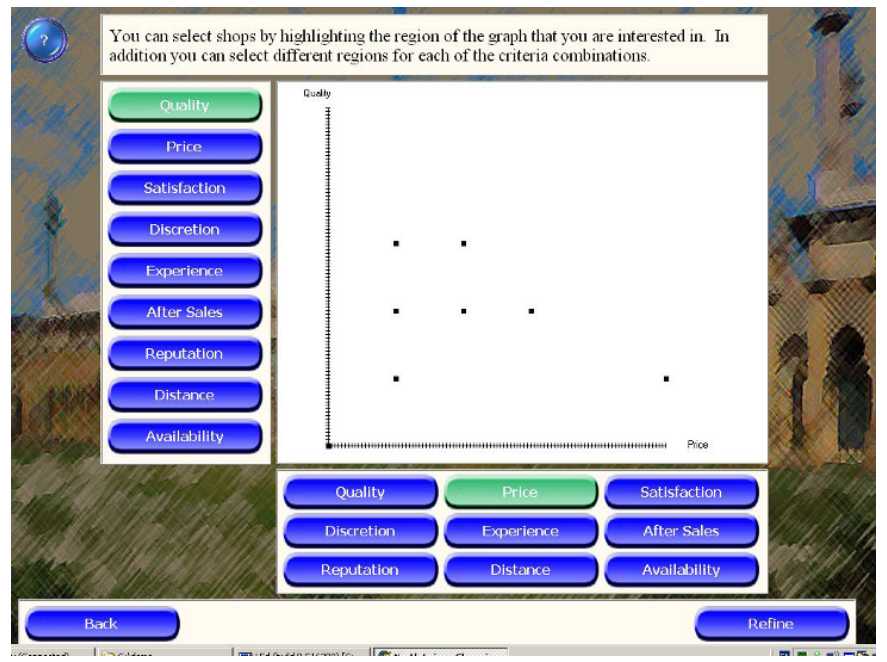

Fig. 4 Advanced Refinement

Another more advanced option for refinement has been provided in which the user is provided with a scatter graph of the shops identified so far. Here, they are able to change the trust attributes represented on the two axes. The user is able to highlight regions of the graph that match the levels and trade-offs between attributes 
which satisfy them. In this way the selection process is customised for each user and various ways of differentiating between results can be explored. For example, figure 4 shows the selection of shops based on a price / quality trade-off of "high price requires higher quality, highest price is limited". One of the aspects of the kiosk we are interested in exploring is how well these methods of refining the model are used by the user. This is discussed in more detail in section 5 .

The feedback stage of the model allows the user to rate each of the trust attributes within the context of their shopping trip. This is accomplished by retrieving the shops details and filling out a small questionnaire where they can rate the attributes and also leave comments. At present we are not directly interested in the issues regarding abusive and malicious behaviour against this functionality, but instead have left this open to be re-addressed at a future date.

Once the user has selected a shop(s) and also possibly those for different types of items, they can then proceed to the route planning aspect of the kiosk. This provides the user with the most efficient path through all of the shops. Internally, a graph based representation of all shop locations based on their GPS coordinates and how they are connected together along the roads in the area, is maintained. When planning the most efficient route, the system will apply an algorithm based on the Traveling Salesman Problem to solve the best route to traverse each of the shops to be visited, just once. Once the path has been calculated it is provided to the user through the use of Google Maps to highlight their journey. On the information gathering and results sections of the kiosk, the user was able to specify the maximum walking time from the initial starting point. This would result in the kiosk precluding any shops that fall outside of this maximum walking distance. The route path would also take this into account, and thus, only the shops within the required walking time/distance would be provided.

\section{Evaluation}

When it came to evaluating the kiosk we used a group of sixteen students that represented the target users, comprising 7 native English speakers and 9 where English was their second language with varying fluency. They each undertook eight tasks that varied in complexity coupled with a follow up questionnaire. A control group of 3 native English speakers undertook the final task of planning a shopping route from an existing web-site.

We used a mock-up kiosk comprising a touch screen monitor on a stand to allow the correct orientation so that users could use it naturally. The eight tasks were designed to indicate how well participants would be able to interact with the kiosk for each function. The tasks were initially oriented towards providing the user with an introduction to each of the different aspects of the system. The final set of tasks dealt with using the system as a whole with the overall goal of defining a shopping route for several items from a varying number of shops. 
When trying to characterise the types of metrics, we drew upon Nicholas et al [4]. Our primary metric was task time, although we had more detailed information through video and application logs which are still being analysed. The mean for each tasks execution time are shown in figure 5.

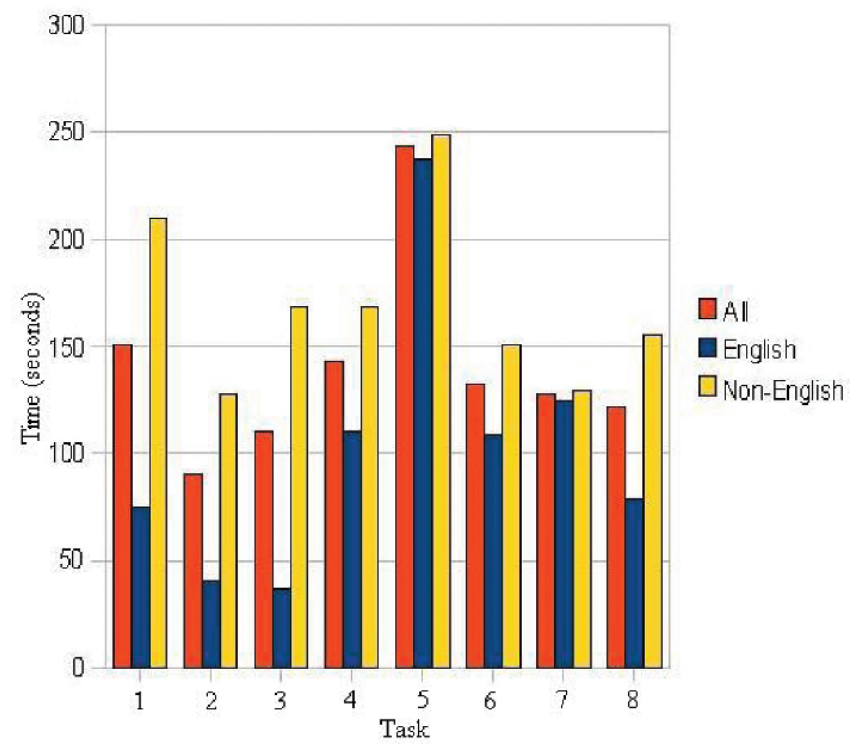

Fig. 5 Task Means

There are a number of points that we can extrapolate out of the evaluation timing results and post-evaluation questionnaire:-

\section{Control Group}

- The control group performed the same final task as the other participants except it was completed using an existing on-line website. Analysis of the group showed that the task completion times were comparable to the primary group of participants. It was noted that due to the limited nature of the interface (a static web page) that participants relied on pen and paper to work out approximate distances and location relationships between each shop. However, this proved to be unsuccessful (within 1 to 2 minutes of starting the task) which resulted in participants selecting the first several shops on each page without applying any thought on the choices made.

2. English and Non-English participants

- We observed that non-native English speakers (students that had only recently begun studying within the UK) had noticeably longer task times than native speakers in the early part of the study, as shown in figure 5. However, as they 
progressed through the study, using more of the features of the system, the times become comparable across these two groups. The times diverged again in the last task, however familiarity with the particular shops was a factor here. So, while there is a learning curve we believe that the concepts are not specific to English culture.

\section{Participants' Opinions:}

- The questionnaire focused on a number of points to gain information regarding the participants user experience. This incorporated asking questions regarding general interface design, and more explicitly, on each of the main areas of the system with respect to the tasks undertaken. With regards to the overall satisfaction of using the kiosk, $13 \%$ said that they were very happy, $81 \%$ said that they were happy while $6 \%$ gave an average score. In addition, when asked if they found choosing shops based on the trust model and subsequent route plan generation helpful, $68.75 \%$ said yes, $25 \%$ said maybe while $6.25 \%$ did not answer the question. Finally, when it came to finding out whether participants would use the kiosk if it was generally available, $68.75 \%$ said yes, $25 \%$ said maybe while $6.25 \%$ did not answer the question.

- From these results we can see that a high proportion of participants found the process of choosing shops based on what was returned from the trust model to be very helpful. We can also establish that the route planning aspect, coupled with the trust model proved to be high favorable between the participants, and given the opportunity, a high proportion would use the system irrespective of the language spoken. In each of these questions, no participant said they did not find the kiosk useful, but instead were all positive.

4. Ease of use:

- When asked to indicate how easy each task was (see table 4) and how intuitive it was to accomplish the task (see table 5).

\begin{tabular}{|c|c|c|c|c|c|}
\hline Task & Very Easy & Easy & Average & Difficult & Very Difficult \\
\hline Basic Refine & $6.25 \%$ & $50 \%$ & $25 \%$ & $18.75 \%$ & $0 \%$ \\
\hline Advanced Refine & $0 \%$ & $12.5 \%$ & $25 \%$ & $50 \%$ & $12.5 \%$ \\
\hline Feedback & $25 \%$ & $50 \%$ & $25 \%$ & $0 \%$ & $0 \%$ \\
\hline
\end{tabular}

Table 4 Ease of use for tasks

\begin{tabular}{|c|c|c|c|c|c|}
\hline Task & Very Intuitive & Intuitive & Average & Bad & Very Bad \\
\hline Basic Refine & $13 \%$ & $50 \%$ & $31 \%$ & $6 \%$ & $0 \%$ \\
\hline Advanced Refine & $0 \%$ & $38 \%$ & $38 \%$ & $6 \%$ & $19 \%$ \\
\hline Feedback & $31 \%$ & $50 \%$ & $13 \%$ & $6 \%$ & $0 \%$ \\
\hline
\end{tabular}

Table 5 Intuitiveness of tasks 
- It can be seen that the basic refine and feedback aspects of the kiosk were easy to use as indicated in the high percentage of positive ratings. This was also reflected in the intuitiveness results for the same operations.

- The advanced refine option (using scatter graphs to refine the trust model) performed poorly as both types of participants found this functionality to be hard to understand with a high percentage of the participants not being able to complete the associated task. This also impacted on its lower intuitiveness scores.

\section{Conclusion}

The purpose of this work was to examine whether trust can be built between a user and third party application through both the interface and underlying trust model.

We have developed a model of consumer trust in shops which condenses the wide range of issues discussed in the literature into a process model and a nine attribute model of trust. We have verified that these attributes are all valid and reflect issues of significance to substantial proportions of our survey population. There was a range of importance attached to each attribute, no one attribute dominating shop selection, i.e. our subjects were not making decisions solely on price or quality etc. Hence, exposing all of our nine attributes allows a proper balance of these factors for a particular user, whereas a summary rating would fail for significant sections of the population.

We then applied this model in developing a kiosk system to support shop selection and route planning. In experiments with a prototype, using trust in selecting a sub-set of shops to visit was found to be quickly understood by all; and as quick as a control system while yielding a more informed selection of shops to visit.

So, we believe that exposing trust as a criteria in shop selection is practical and natural. We are further developing our application for a larger study.

\subsection{Acknowledgments}

Thanks to the Network Lab group for their help in participating in different studies and reviewing the paper. Also, thanks goes out to the participants of the questionnaires and evaluations.

\section{References}

1. G. Lancaster, L. Massingham, Essentials of Marketing, ISBN 0070841810, 1988.

2. S.C. Chen, G.S. Dhillon, Interpreting Dimensions of Consumer Trust in E-Commerce, Journal of Information Technology and Management, Vol 4 (2), pp 303-318, 2003. 
3. D.H. McKnight, V. Choudhury, C. Kacmar, The impact of initial consumer trust on intentions to transact with a web site: a trust building model, Journal of Strategic Information Systems, Vol 11 (3-4), pp 297-323, 2002.

4. D. Nicholas, P. Huntington, P. Williams, P., Establishing metrics for the evaluation of touch screen kiosks, Journal of Information Science, Vol 27(2), pp 61-71, 2001.

5. F.N. Egger, F.N., 'Trust me, I'm an online vendor": towards a model of trust for e-commerce system design, Conference on Human Factors in Computing Systems, pp 101-102, 2000.

6. M.S. Kim, J.H. Ahn, A model for buyer's trust in the e-marketplace, Proceedings of the 7th international conference on Electronic commerce, pp 195-200, 2005.

7. J.A. Howard, J.N. Sheth, The theory of buyer behavior, Wiley, 1969.

8. J. Riegelsberger, M.A. Sasse, "Trust Me, I'ma. com": Reassuring shoppers in electronic retail environments, Intermedia, 2000.

9. J. Riegelsberger, M.A. Sasse, J.D. McCarthy, The mechanics of trust: A framework for research and design, International Journal of Human-Computer Studies, Vol 62 (3), pp 381-422, 2005.

10. M. Lee, E. Turban, A Trust Model for Consumer Internet Shopping, International Journal of Electronic Commerce, Vol 6(1), pp 75-91, 2001.

11. F.E. Webster, Y. Wind, A general model for understanding organizational buying behaviour, Journal of marketing, Vol 36, pp 12-19, 1972.

12. J.N. Sheth, A model of Industrial Buyer Behaviour, Journal of Marketing, Vol 37, pp 50-56, 1973.

13. J.N. Sheth, "A theory of family buying decisions", in Sheth, J.N. (Eds),Models of Buyer Behavior, Harper \& Row, New York, NY, pp.17-33, 1974.

14. J. Engel, R.D. Blackwell, D. Kollat, Consumer Behaviour, 3rd edition, New York: Holt, Rinehart, and Winston, 1979.

15. Hardens London Resturant Guide, www.hardens.com, 2007. 